\title{
Subgradients of value functions in parametric dynamic programming *
}

\author{
B. T. Kien ${ }^{\dagger}$ Y. C. Liou ${ }^{\ddagger}$ N.-C. Wong ${ }^{\S}$ and J.-C. Yao
}

May 21, 2007

\begin{abstract}
We study in this paper the first-order behavior of value functions in parametric dynamic programming with linear constraints and nonconvex cost functions. By establishing an abstract result on the Fréchet subdifferential of value functions of parametric mathematical programming problems, some new formulas on the Fréchet subdifferential of value functions in parametric dynamic programming are obtained.
\end{abstract}

Key words: Dynamic programming, value functions, Fréchet nomal cones, Fréchet subgradients, the Fréchet subdifferential.

\footnotetext{
${ }^{*}$ This research was partially supported by a grant from National Science Council of Taiwan, ROC.

†Department of Applied Mathematics, National Sun Yat-Sen University, Kaohsiung, Taiwan 804 (on leave from National University of Civil Engineering, 55 Giai Phong, Hanoi, Vietnam); email: btkien@math.nsysu.edu.tw

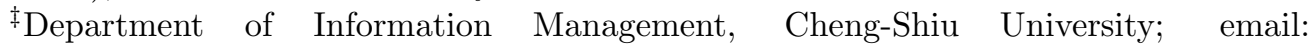
ycliou@csu.edu.tw.

$\S$ Department of Applied Mathematics, National Sun Yat-Sen University, Kaohsiung, Taiwan 804; email: wong@math.nsysu.edu.tw.

"Corresponding author. Department of Applied Mathematics, National Sun Yat-Sen University, Kaohsiung, Taiwan 804; email: yaojc@math.nsysu.edu.tw.
} 


\section{Introduction}

A wide variety of problems in discrete optimal control can be posed in the following form.

Determine a control vector $u=\left(u_{0}, u_{1}, \ldots, u_{N-1}\right) \in U_{0} \times U_{1} \times \cdots \times U_{N-1}$ and a path $x=\left(x_{0}, x_{1}, \ldots, x_{N}\right) \in X_{0} \times X_{1} \times \cdots \times X_{N}$ which minimize the cost

$$
\sum_{k=0}^{N-1} h_{k}\left(x_{k}, u_{k}, w_{k}\right)+h_{N}\left(x_{N}\right)
$$

with state equation

$$
x_{k+1}=A_{k} x_{k}+B_{k} u_{k}+T_{k} w_{k}, \text { for all } k=0,1, \ldots, N-1,
$$

the constraints

$$
u_{k} \in \Omega_{k} \subset X_{k} \text { for all } k=0,1, \ldots, N-1
$$

and initial condition

$$
x_{0}=x \in X_{0},
$$

where

$k$ indexes discrete time,

$x_{k}$ is the state of the system and summarizes past information that is relevant for future optimization,

$u_{k}$ is the control variable to be selected at time $k$ with knowledge of the state $x_{k}$,

$w_{k}$ is a random parameter (also called disturbance or noise),

$N$ is the horizon or number times control is applied,

$X_{k}$ is a finite-dimensional space of state variables at stage $k$,

$U_{k}$ is a finite-dimensional space of control variables at stage $k$,

$\Omega_{k}$ is a nonempty set in $U_{k}$.

$W_{k}$ is a finite-dimensional space of random parameters at stage $k$. 
A classical example for the problem (1)-(4) is the inventory control problem where $x_{k}$ plays a stock available at the beginning of the $k$ th period; $u_{k}$ plays a stock order at the beginning of the $k$ th period and $w_{k}$ is the demand during the $k$ th period with given probability distribution; and the cost function has the form $\Sigma_{k=0}^{N-1} c u_{k}+H\left(x_{k}+u_{k}-w_{k}\right)$ together with state equation $x_{k+1}=x_{k}+u_{k}-w_{k}$ (see [3] for details).

Put $W=W_{0} \times W_{1} \times \cdots \times W_{N-1}, X=X_{0} \times X_{1} \times \cdots \times X_{N}$ and $U=$ $U_{0} \times U_{1} \times \cdots \times U_{N-1}$. We denote by $V(w)$ the optimal value of the problem (1)-(4) corresponding to the parameter $w=\left(w_{0}, w_{1}, \ldots, w_{N-1}\right) \in W$. Thus $V: W \rightarrow \bar{R}$ is an extended real-valued function which is called the value function of the problem (1)-(4).

The study of first-oder behavior of value functions is of importance in analysis and optimization. An example of this type is distance functions and its applications in optimal control problems with differential inclusions (e.g., [1], [8], [26]). There have been many papers dealt with differentiability properties and the Fréchet subdifferential of value functions in the literature (e.g., [6], [16], [18], [21]). Under Lipschitzian conditions and the assumption that the solution set of perturbed problems is nonempty in a neighborhood of a unperturbed problem, Clarke [6, Theorem 6.52] established a useful formula for the generalized gradient of value function. By considering a set of assumptions which involves a kind of coherence property, Penot [21] showed that the value functions are Fréchet differentiable. The results of Penot gave sufficient conditions under which the value functions are Fréchet differentiable rather than formulas computing their derivatives.. In [16], Mordukhovich, Nam and Yen derived formulas for computing and estimating the so-called Fréchet suddiferential of value functions of parametric mathematical programming problems in Banach spaces without Lipschitzian assumptions.

Beside the study of first-oder behavior of value functions in parametric mathematical programming, the study of first-oder behavior of value functions in optimal control is also of interest especially because of potential connections with feedback rules as well as Hamilton-Jacobi-Bellman equations. We refer the reader to [20], [22], [24] and [25] for recent studies on sensitivity analysis of the optimal-value function in parametric optimal control. In particular, Seeger [24] obtained a formula for the approximate subdifferential of convex analysis of $V$ to the case where $h_{k}$ and $\Omega_{k}$ were assumed to be con- 
vex, and the problem can have no optimal paths. It is noted that if $\Omega_{k}$ and $h_{k}$ are convex for all $k=0,1, \ldots, N$, then $V$ becomes a convex function. In this case, we can compute the subdifferential of $V$ via subgradients of convex functions. However, the situation will be more complicated if $h_{k}$ and $\Omega_{k}$ are not convex because subgradients of convex functions fail to apply.

It is well recognized that the function $V$ can fail to be smooth despite any degree of smoothness of $h_{k}$. The aim of this paper is to derive some new formula for computing the so-called Fréchet subdifferential of $V$ via the tool of generalized differentiation. In order to obtain the result, we first establish a formula for computing and estimating the Fréchet subdifferential of the value functions for a special class of parametric mathematical programming problems (Theorem 2.1). Then we apply Theorem 2.1 to prove Theorem 1.1 which is the main result of this paper. Our proof of Theorem 2.1 closely follows the method of [16]. However, we deal with the formula of basic normals to set intersections in the product of Asplund spaces and establish a formula for computing the normal cone of contraint sets.

Let us recall some notions related to generalized differentiation. The notions and results of generalized differentiation can be found in [14] and [15]. Let $\varphi: Z \rightarrow \bar{R}$ be a extended-real-valued function on a finite dimensional space $Z$ and $\bar{x} \in Z$ be such that $\varphi(\bar{x})$ is finite. The set

$$
\hat{\partial} \varphi(\bar{x}):=\left\{x^{*} \in X \mid \liminf _{x \rightarrow \bar{x}} \frac{\varphi(x)-\varphi(\bar{x})-\left\langle x^{*}, x-\bar{x}\right\rangle}{\|x-\bar{x}\|} \geq 0\right\}
$$

is called the Fréchet subdifferential of $\varphi$ at $\bar{x}$. A vector $x^{*} \in \widehat{\partial} \varphi(\bar{x})$ is called a Fréchet subgradient of $\varphi$ at $\bar{x}$. It is known that the Fréchet subdifferential reduces to the classical Fréchet derivative for differentiable functions and to subdifferential of convex analysis for convex functions. The set $\hat{\partial}^{+} \varphi(\bar{x}):=$ $-\hat{\partial}(-\varphi)(\bar{x})$ is called the upper subdifferential of $\varphi$ at $\bar{x}$.

Let $\Omega$ be a nonempty set in $Z$. Given $\bar{z} \in \Omega$ and $\epsilon \geq 0$, define the set of $\epsilon$-normal by

$$
\widehat{N}_{\epsilon}(\bar{z} ; \Omega):=\left\{z^{*} \in Z^{*} \mid \limsup _{z \rightarrow \bar{z}} \frac{\left\langle z^{*}, z-\bar{z}\right\rangle}{\|z-\bar{z}\|} \leq \epsilon\right\} .
$$

When $\epsilon=0$, the set $\widehat{N}(\bar{z} ; \Omega):=\widehat{N}_{0}(\bar{z} ; \Omega)$ is called the Fréchet normal cone to $\Omega$ at $\bar{z}$. It is also well known that if $\delta(z, \Omega)$ is the indicator function of $\Omega$, i.e., 
$\delta(z, \Omega):=0$ if $z \in \Omega$ and $\delta(z, \Omega):=\infty$ otherwise, then $\widehat{N}(\bar{z} ; \Omega)=\widehat{\partial} \delta(\bar{z} ; \Omega)$. A vector $z^{*} \in Z^{*}$ (the dual space of $Z$ ) is called a limiting normal to $\Omega$ at $\bar{z}$ if there exist sequences $\epsilon_{k} \rightarrow 0^{+}, z_{k} \rightarrow \bar{z}$, and $z_{k}^{*} \rightarrow z^{*}$ such that $z_{k}^{*} \in \widehat{N}_{\epsilon_{k}}\left(\bar{z}_{k} ; \Omega\right)$. The collection of such normals is called the Mordukhovich normal cone to $\Omega$ at $\bar{z}$ and is denoted by $N(\bar{z} ; \Omega)$. The set $\Omega$ is said to be normally regular at $z \in \Omega$ (in the sense of [14]) if $\widehat{N}(z ; \Omega)=N(z ; \Omega)$. It is also well known that if $\Omega$ is locally convex at $z$, then it is normally regular at $z$ (see $[14, \operatorname{Pr} .1 .5])$.

Let $F: Z \rightrightarrows E$ be a set-valued mapping from $Z$ to a finite-dimensional space $E$ with the domain

$$
\operatorname{dom} F:=\{z \in Z \mid F(z) \neq \varnothing\}
$$

and the graph

$$
\operatorname{gph} F:=\{(z, v) \in Z \times E \mid v \in F(z)\} .
$$

We say that the mapping $F$ admits a locally upper Lipschitzian selection at $(\bar{z}, \bar{v}) \in \operatorname{gph} F$ if there is a single-valued mapping $h: Z \rightarrow E$, which is locally upper Lipschitzian at $\bar{z}$, i.e., there exist numbers $\eta>0$ and $l>0$ such that for any $z \in Z$ with $\|z-\bar{z}\|<\eta$, we have

$$
\|h(z)-h(\bar{z})\| \leq l\|z-\bar{z}\|
$$

which satisfies $h(\bar{z})=\bar{v}$ and $h(z) \in F(z)$ for all $z$ in a neighborhood of $\bar{z}$.

We now return to the problem (1)-(4). For each $w=\left(w_{0}, w_{1}, \ldots, w_{N-1}\right) \in$ $W$ we put

$$
\begin{gathered}
f(x, u, w)=\sum_{k=0}^{N-1} h_{k}\left(x_{k}, u_{k}, w_{k}\right)+h_{N}\left(x_{N}\right), \\
G(w)=\left\{(x, u) \in X \times U \mid x_{k+1}=A_{k} x_{k}+B_{k} u_{k}+T_{k} w_{k}, \quad \forall k=0,1, \ldots, N-1\right\}
\end{gathered}
$$

and $\Omega=\Omega_{0} \times \Omega_{1} \times \cdots \times \Omega_{N-1}$. Then the problem (1)-(4) can be formulated in the following simpler form

$$
V(w):=\inf _{(x, u) \in G(w) \cap(X \times \Omega)} f(x, u, w) .
$$


We denote by $S(w)$ the solution set of the problem (1)-(4) corresponding to the parameter $w \in W$. It is also assumed that $(\bar{x}, \bar{u})$ is a solution of the problem at $\bar{w}$, that is $(\bar{x}, \bar{u}) \in S(\bar{w})$ where $\bar{x}=\left(\bar{x}_{0}, \bar{x}_{1}, \ldots, \bar{x}_{N}\right), \bar{u}=$ $\left(\bar{u}_{0}, \bar{u}_{1}, \ldots, \bar{u}_{N-1}\right)$ and $\bar{w}=\left(\bar{w}_{0}, \bar{w}_{1}, \ldots, \bar{w}_{N-1}\right)$.

We are now ready to state our main result.

Theorem 1.1 Let the value function $V$ defined by (9) be finite at some $\bar{w} \in \operatorname{dom} S, h_{N}$ be Fréchet differentiable at $\bar{x}_{N}$ and $h_{k}$ be Fréchet differentiable at $\left(\bar{x}_{k}, \bar{u}_{k}, \bar{w}_{k}\right)$ for $k=0, \ldots, N-1$. Assume that $T_{k}$ is surjective and $\Omega_{k}$ are normally regular at $\bar{u}_{k}$ for all $k=0,1, \ldots, N-1$. Then for $w^{*}=\left(w_{0}^{*}, w_{1}^{*}, \ldots, w_{N-1}^{*}\right) \in W$ to be a Fréchet subgradient of $V$ at $\bar{w}=$ $\left(\bar{w}_{0}, \bar{w}_{1}, \ldots, \bar{w}_{N-1}\right)$, it is necessary that there exist $u^{*}=\left(u_{0}^{*}, u_{1}^{*}, \ldots, u_{N-1}^{*}\right) \in$ $\widehat{N}(\bar{u} ; \Omega)$ and $z^{*}=\left(z_{1}^{*}, z_{2}^{*}, \ldots, z_{N}^{*}\right) \in X_{1} \times X_{2} \times \cdots \times X_{N}$ such that

$$
\left\{\begin{array}{l}
w_{k}^{*}=\left(\frac{\partial h_{k}}{\partial w_{k}}\right)\left(\bar{x}_{k}, \bar{u}_{k}, \bar{w}_{k}\right)+T_{k}^{*} z_{k+1}^{*} \text { for } k=0,1, \ldots, N-1 . \\
\left(\frac{\partial h_{0}}{\partial x_{0}}\right)\left(\bar{x}_{0}, \bar{u}_{0}, \bar{w}_{0}\right)=-A_{0}^{*} z_{1}^{*},\left(\frac{\partial h_{N}}{\partial x_{N}}\right)\left(\bar{x}_{N}\right)=z_{N}^{*}, \\
\left(\frac{\partial h_{k}}{\partial x_{k}}\right)\left(\bar{x}_{k}, \bar{u}_{k}, \bar{w}_{k}\right)=z_{k}^{*}-A_{k}^{*} z_{k+1}^{*} \text { for } k=1,2, \ldots, N-1 \\
\left(\frac{\partial h_{k}}{\partial u_{k}}\right)\left(\bar{x}_{k}, \bar{u}_{k}, \bar{w}_{k}\right)=-u_{k}^{*}-B_{k}^{*} z_{k+1}^{*} \text { for } k=0,1, \ldots, N-1 .
\end{array}\right.
$$

The above condition is also sufficient for $w^{*} \in \widehat{\partial} V(\bar{w})$ if the solution map $S$ has an upper Lipschitzian selection at $(\bar{w}, \bar{x}, \bar{u})$.

When $\bar{u}_{k} \in \operatorname{int} \Omega_{k}$ (the interior of $\Omega_{k}$ ) or $\Omega_{k}=U_{k}$ for $k=0,1, \ldots, N-1$, one has $\widehat{N}\left(\bar{u}_{k} ; \Omega_{k}\right)=\{0\}$. In this case we have the following result.

Corollary 1.1 Assume assumptions as in Theorem 1.1 and $\widehat{N}(\bar{u} ; \Omega)=\{0\}$. Then for $w^{*}=\left(w_{0}^{*}, w_{1}^{*}, \ldots, w_{N-1}^{*}\right) \in W$ to be a Fréchet subgradient of $V$ at $\bar{w}=\left(\bar{w}_{0}, \bar{w}_{1}, \ldots, \bar{w}_{N-1}\right)$, it is necessary that there exists $z^{*}=\left(z_{1}^{*}, z_{2}^{*}, \ldots, z_{N}^{*}\right) \in$ $X_{1} \times X_{2} \times \cdots \times X_{N}$ such that

$$
\left\{\begin{array}{l}
w_{k}^{*}=\left(\frac{\partial h_{k}}{\partial w_{k}}\right)\left(\bar{x}_{k}, \bar{u}_{k}, \bar{w}_{k}\right)+T_{k}^{*} z_{k+1}^{*} \text { for } k=0,1, \ldots, N-1 . \\
\left(\frac{\partial h_{0}}{\partial x_{0}}\right)\left(\bar{x}_{0}, \bar{u}_{0}, \bar{w}_{0}\right)=-A_{0}^{*} z_{1}^{*},\left(\frac{\partial h_{N}}{\partial x_{N}}\right)\left(\bar{x}_{N}\right)=z_{N}^{*}, \\
\left(\frac{\partial h_{k}}{\partial x_{k}}\right)\left(\bar{x}_{k}, \bar{u}_{k}, \bar{w}_{k}\right)=z_{k}^{*}-A_{k}^{*} z_{k+1}^{*} \text { for } k=1,2, \ldots, N-1 \\
\left(\frac{\partial h_{k}}{\partial u_{k}}\right)\left(\bar{x}_{k}, \bar{u}_{k}, \bar{w}_{k}\right)=-B_{k}^{*} z_{k+1}^{*} \text { for } k=0,1, \ldots, N-1 .
\end{array}\right.
$$


The above condition is also sufficient for $w^{*} \in \widehat{\partial} V(\bar{w})$ if the solution map $S$ has an upper Lipschitzian selection at $(\bar{w}, \bar{x}, \bar{u})$.

In order to prove Theorem 1.1, we shall need a result on the Fréchet subdifferential of the value functions from a abstract model which is contained in the next section.

\section{A result on the Fréchet subdifferential of value functions}

Let $X, Y$ and $Z$ be finite-dimensional spaces. Assume that $A: X \rightarrow Z$ and $T: Y \rightarrow Z$ are linear mappings. Let $A^{*}: Z \rightarrow X$ and $T^{*}: Z \rightarrow Y$ be adjoint mappings of $A$ and $T$, respectively. Let $f: X \times Y \rightarrow \bar{R}$ be a function. For each $w \in Y$, we put

$$
G(w):=\{x \in X \mid A x=T w\} .
$$

Let $\bar{w} \in Y$ and $K$ be a nonempty set in $X$ such that $K \cap G(w) \neq \varnothing$ for all $w$ in a neighborhood of $\bar{w}$. Consider the problem

$$
\mu(w):=\inf _{x \in G(w) \cap K} f(x, w) .
$$

We denote by $M(w)$ the solution set of the problem (11) corresponding to the parameter $w \in Y$. Assume that $\bar{x}$ is a solution of the problem (11) corresponding to the parameter $\bar{w}$, i.e., $\bar{x} \in M(\bar{w})$.

The following result gives a formula computing the Fréchet subdifferential of $\mu$ at $\bar{w}$.

Theorem 2.1 Let the value function $\mu$ defined by (11) be finite at $\bar{w} \in$ $\operatorname{dom} M$, and let $\bar{x} \in M(\bar{w})$ be such that $\hat{\partial}^{+} f(\bar{x}, \bar{w}) \neq \varnothing$. Assume that $\operatorname{im} A \subset$ $\operatorname{im} T$ and $K$ is normally regular at $\bar{x}$. Then one has

$$
\widehat{\partial} \mu(\bar{w}) \subset \bigcap_{\left(x^{*}, v^{*}\right) \in \hat{\partial}^{+} f(\bar{x}, \bar{w})} \bigcup_{u^{*} \in \widehat{N}(\bar{x} ; K)}\left[v^{*}+T^{*}\left(\left(A^{*}\right)^{-1}\left(x^{*}+u^{*}\right)\right)\right] .
$$


If in addition, $f$ is Fréchet differentiable at $(\bar{x}, \bar{w})$ and the solution map $M$ has an upper Lipschitzian selection at $(\bar{w}, \bar{x})$, then

$$
\widehat{\partial} \mu(\bar{w})=\bigcup_{u^{*} \in \widehat{N}(\bar{x} ; K)}\left[\nabla_{w} f(\bar{x}, \bar{w})+T^{*}\left(\left(A^{*}\right)^{-1}\left(\nabla_{x} f(\bar{x}, \bar{w})+u^{*}\right)\right)\right] .
$$

To prove Theorem 2.1, we need the following lemmas.

Lemma 2.1 Assume that $\operatorname{im} A \subset \operatorname{im} T$ and $(\bar{w}, \bar{x}) \in g p h G$. Then one has

$$
\widehat{N}((\bar{w}, \bar{x}) ; \operatorname{gph} G)=\left\{\left(-T^{*} z^{*}, A^{*} z^{*}\right) \mid z^{*} \in Z\right\} .
$$

Proof. Since gph $G$ is a closed convex set, the Fréchet normal cone $\widehat{N}((\bar{w}, \bar{x})$; gph $G)$ coincides with the normal cone in the sense of convex analysis. For the convenience we put $Q=\operatorname{gph} G$. We now claim that if $\left(w^{*}, x^{*}\right) \in N_{Q}(\bar{w}, \bar{x})$ which is the normal cone to $Q$ at $(\bar{w}, \bar{x})$ in the sense of convex analysis, then $\left(-w^{*}, x^{*}\right) \in \operatorname{im} T^{*} \times \operatorname{im} A^{*}$. On the contrary, suppose that $\left(-w^{*}, x^{*}\right) \notin \operatorname{im} T^{*} \times \operatorname{im} A^{*}$. By the separation theorem for convex sets, there exists $\left(w_{0}, x_{0}\right) \in Y \times X$ such that

$$
\left\langle\left(-w^{*}, x^{*}\right),\left(w_{0}, x_{0}\right)\right\rangle>\left\langle\left(T^{*} u^{*}, A^{*} u^{*}\right),\left(w_{0}, x_{0}\right)\right\rangle \forall u^{*} \in Y .
$$

This is equivalent to

$$
-\left\langle w^{*}, w_{0}\right\rangle+\left\langle x^{*}, x_{0}\right\rangle>\left\langle u^{*}, T w_{0}+A x_{0}\right\rangle \forall u^{*} \in Y .
$$

Hence $\left\langle x^{*}, x_{0}\right\rangle-\left\langle w^{*}, w_{0}\right\rangle>0$ and $T w_{0}+A x_{0}=0$. Putting $w_{0}^{\prime}=-w_{0}$, one has $\left(w_{0}^{\prime}, x_{0}\right) \in Q$ and $\left\langle x^{*}, x_{0}\right\rangle+\left\langle w^{*}, w_{0}^{\prime}\right\rangle>0$. Since $\left(w^{*}, x^{*}\right) \in N_{Q}(\bar{w}, \bar{x})$,

$$
\left\langle\left(w^{*}, x^{*}\right),(w-\bar{w}, x-\bar{x})\right\rangle \leq 0 \forall(w, x) \in Q .
$$

Putting $x_{t}=\bar{x}+t x_{0}$ and $w_{t}=\bar{w}+t w_{0}^{\prime}$, we have $\left(w_{t}, x_{t}\right) \in Q$ for all $t>0$. Substituting $\left(w_{t}, x_{t}\right)$ into (15) yields

$$
\left\langle w^{*}, t w_{0}^{\prime}\right\rangle+\left\langle x^{*}, t x_{0}\right\rangle \leq 0 .
$$

This implies

$$
\left\langle w^{*}, w_{0}^{\prime}\right\rangle+\left\langle x^{*}, x_{0}\right\rangle \leq 0,
$$


which contradicts the fact $\left\langle x^{*}, x_{0}\right\rangle+\left\langle w^{*}, w_{0}^{\prime}\right\rangle>0$ derived above. Thus our claim is proved.

Take any $\left(w^{*}, x^{*}\right) \in N_{Q}(\bar{w}, \bar{x})$. By the claim established above, there exists $z^{*} \in Z^{*}$ such that $-w^{*}=T^{*} z^{*}$. By the definition of the normal cone, we have

$$
\left\langle\left(-T^{*} z^{*}, x^{*}\right),(w-\bar{w}, x-\bar{x})\right\rangle \leq 0 \forall(w, x) \in Q .
$$

This is equivalent to

$$
\left.\left\langle-z^{*}, T w-T \bar{w}\right\rangle+\left\langle x^{*}, x-\bar{x}\right)\right\rangle \leq 0 \forall(w, x) \in Q .
$$

Since $\operatorname{im} A \subset \operatorname{im} T$, for any $x \in X$ there exists $w \in Y$ such that $T w=A x$. Putting $x_{t}=\bar{x}+t x$ and $w_{t}=\bar{w}+t w$, we see that $\left(w_{t}, x_{t}\right) \in Q$ for all $t>0$. Substituting $\left(w_{t}, x_{t}\right)$ into (16), we have

$$
\left\langle-z^{*}, t T w\right\rangle+\left\langle x^{*}, t x\right\rangle \leq 0
$$

which implies that

$$
\left\langle-A^{*} z^{*}, x\right\rangle+\left\langle x^{*}, x\right\rangle \leq 0 .
$$

Since $x$ is arbitrary, $x^{*}=A^{*} z^{*}$. Conversely, assume that $\left(w^{*}, x^{*}\right)=$ $\left(-T^{*} z^{*}, A^{*} z^{*}\right)$ for some $z^{*} \in Z$. If $\left(w^{*}, x^{*}\right) \notin N_{Q}(\bar{w}, \bar{x})$, then there exists $(w, x) \in Q$ such that

$$
\left\langle\left(-T^{*} z^{*}, A^{*} z^{*}\right),(w-\bar{w}, x-\bar{x})\right\rangle>0
$$

which is equivalent to

$$
\left\langle-z^{*}, T w-T \bar{w}\right\rangle+\left\langle z^{*}, A x-A \bar{x}\right\rangle>0 .
$$

Hence

$0=\left\langle-z^{*}, A x-A \bar{x}\right\rangle+\left\langle z^{*}, A x-A \bar{x}\right\rangle=\left\langle-z^{*}, T w-T \bar{w}\right\rangle+\left\langle z^{*}, A x-A \bar{x}\right\rangle>0$,

which is absurd. Therefore $\left(w^{*}, x^{*}\right) \in N_{Q}(\bar{w}, \bar{x})$ and the proof of the lemma is complete.

Lemma 2.2 Let $P=Y \times K$ and $Q=\operatorname{gph} G$. Assume that $(\bar{w}, \bar{x}) \in P \cap Q$ and $K$ is normally regular at $\bar{x}$. Then one has

$$
\widehat{N}((\bar{w}, \bar{x}) ; P \cap Q)=\{0\} \times \widehat{N}(\bar{x} ; K)+\widehat{N}((\bar{w}, \bar{x}) ; Q) .
$$


Proof. we note that

$$
\widehat{N}((\bar{w}, \bar{x}) ; P)=\widehat{N}(\bar{w} ; Y) \times \widehat{N}(\bar{x} ; K)=\{0\} \times \widehat{N}(\bar{x} ; K) .
$$

Hence the set $P$ is also normally regular at $(\bar{w}, \bar{x})$. Since $Q$ is convex, it is normal regular at $(\bar{w}, \bar{x})$. It remains to show that the system sets $\{P, Q\}$ satisfies the normal qualification condition, that is,

$$
\widehat{N}((\bar{w}, \bar{x}) ; Q) \cap[-\widehat{N}((\bar{w}, \bar{x}) ; P)]=\{(0,0)\} .
$$

Take any $\left(w^{*}, x^{*}\right) \in \widehat{N}((\bar{w}, \bar{x}) ; Q) \cap[-\widehat{N}((\bar{w}, \bar{x}) ; P)]$. Then we have $w^{*}=0$, $-x^{*} \in \widehat{N}(\bar{x} ; K)$ and $w^{*}=-T^{*} z^{*}, x^{*}=A^{*} z^{*}$ for some $z^{*} \in Z$. If $x^{*} \neq 0$, then there exists $x \in X$ such that $\left\langle x^{*}, x\right\rangle=\left\langle A^{*} z^{*}, x\right\rangle \neq 0$. Hence $\left\langle z^{*}, A x\right\rangle \neq 0$. Since $\operatorname{im} A \subset \operatorname{im} T$, there exists $w \in Y$ such that $A x=T w$. Consequently, we have

$$
0 \neq\left\langle z^{*}, A x\right\rangle=\left\langle z^{*}, T w\right\rangle=\left\langle T^{*} z^{*}, w\right\rangle=0,
$$

which is absurd. Thus we must have $x^{*}=0$ and so the normal qualification condition is satisfied. According to Theorem 3.4 in [14], we have

$$
\widehat{N}((\bar{w}, \bar{x}) ; P \cap Q)=\{0\} \times \widehat{N}(\bar{x} ; K)+\widehat{N}((\bar{w}, \bar{x}) ; Q) .
$$

The proof of the lemma is now complete.

Proof of Theorem 2.1. In the arguments below, we will use some techniques from [16].

Take any $w^{*} \in \hat{\partial} \mu(\bar{w})$. Then for any $\epsilon>0$, there exists a neighborhood $U$ of $\bar{w}$ such that

$$
\left\langle w^{*}, w-\bar{w}\right\rangle \leq \mu(w)-\mu(\bar{w})+\epsilon\|w-\bar{w}\| \forall w \in U .
$$

Hence

$$
\left\langle w^{*}, w-\bar{w}\right\rangle \leq f(x, w)-f(\bar{x}, \bar{w})+\epsilon\|w-\bar{w}\|
$$

for all $w \in U$ and $x \in G(w) \cap K$. Taking any $\left(x^{*}, v^{*}\right) \in \hat{\partial}^{+} f(\bar{x}, \bar{w})$, we have $-\left(x^{*}, v^{*}\right) \in \hat{\partial}(-f)(\bar{x}, \bar{w})$. By Theorem 1.88 in [14], there exists a function $s: X \times Y \rightarrow R$ that is finite around $(\bar{x}, \bar{w})$, Fréchet differentiable at $\bar{w}$ and satisfies the following relations:

$s(\bar{x}, \bar{w})=f(\bar{x}, \bar{w}), \nabla s(\bar{x}, \bar{w})=\left(x^{*}, v^{*}\right)$, and $s(x, w) \geq f(x, w) \forall(x, w) \in X \times Y$. 
From the above relations and (17), we get

$$
\left\langle w^{*}, w-\bar{w}\right\rangle \leq s(x, w)-s(\bar{x}, \bar{w})+\epsilon\|w-\bar{w}\| \forall w \in U, x \in G(w) \cap K .
$$

By using the Taylor expansion, (18) implies

$\left\langle w^{*}, w-\bar{w}\right\rangle \leq\langle\nabla s(\bar{x}, \bar{w}),(x-\bar{x}, w-\bar{w})\rangle+o(\|x-\bar{x}\|+\|w-\bar{w}\|)+\epsilon\|w-\bar{w}\|$

for all $w \in U$ and $x \in G(w) \cap K$. This is equivalent to saying that

$$
\left\langle w^{*}-v^{*}, w-\bar{w}\right\rangle-\left\langle x^{*}, x-\bar{x}\right\rangle \leq o(\|x-\bar{x}\|+\|w-\bar{w}\|)+\epsilon\|w-\bar{w}\|
$$

for all $(w, x) \in(U \times K) \cap Q$.

Since $\epsilon$ was chosen arbitrarily, it yields

$$
\limsup _{(w, x) \stackrel{P \cap Q}{\longrightarrow}(\bar{w}, \bar{x})} \frac{\left\langle\left(w^{*}-v^{*},-x^{*}\right),(w, x)-(\bar{w}, \bar{x})\right\rangle}{\|w-\bar{w}\|+\|x-\bar{x}\|} \leq 0,
$$

from which it follows that

$$
\left(w^{*}-v^{*},-x^{*}\right) \in \widehat{N}((\bar{w}, \bar{x}) ; P \cap Q) .
$$

By Lemma 2.2,

$$
\left(w^{*}-v^{*},-x^{*}\right) \in\{0\} \times \widehat{N}(\bar{x} ; K)+\widehat{N}((\bar{w}, \bar{x}) ; Q) .
$$

Hence there exists $u^{*} \in \widehat{N}(\bar{x} ; K)$ such that $\left(w^{*}-v^{*},-x^{*}-u^{*}\right) \in \widehat{N}((\bar{w}, \bar{x}) ; Q)$. By Lemma 2.1, there exists $z^{*} \in Y$ such that $w^{*}-v^{*}=-T^{*} z^{*}$ and $-x^{*}-u^{*}=$ $A^{*} z^{*}$. This implies that $w^{*}=v^{*}+T^{*}\left(-z^{*}\right)$ and $-z^{*} \in\left(A^{*}\right)^{-1}\left(x^{*}+u^{*}\right)$. Consequently, $w^{*} \in v^{*}+T^{*}\left[\left(A^{*}\right)^{-1}\left(x^{*}+u^{*}\right)\right]$ and so we obtain the first assertion.

In order to prove the second assertion, it is sufficient to show that

$$
\widehat{\partial} \mu(\bar{w}) \supset \bigcup_{u^{*} \in \widehat{N}(\bar{x} ; K)}\left[\nabla_{w} f(\bar{x}, \bar{w})+T^{*}\left(\left(A^{*}\right)^{-1}\left(\nabla_{x} f(\bar{x}, \bar{w})+u^{*}\right)\right)\right] .
$$

On the contrary, suppose that there exists $w^{*} \in Y$ such that

$$
w^{*} \in\left(\bigcup_{u^{*} \in \widehat{N}(\bar{x} ; K)}\left[\nabla_{w} f(\bar{x}, \bar{w})+T^{*}\left(\left(A^{*}\right)^{-1}\left(\nabla_{x} f(\bar{x}, \bar{w})+u^{*}\right)\right)\right]\right) \backslash \widehat{\partial} \mu(\bar{w}) .
$$


Then we can find an $\bar{\epsilon}>0$ and a sequence $w_{k} \rightarrow \bar{w}$ such that

$$
\left\langle w^{*}, w_{k}-\bar{w}\right\rangle>\mu\left(w_{k}\right)-\mu(\bar{w})+\bar{\epsilon}\left\|w_{k}-\bar{w}\right\| .
$$

Let $h$ be an upper Lipschitzian selection of the solution map $M$. Putting $x_{k}=h\left(w_{k}\right)$, we have $x_{k} \in M\left(w_{k}\right)$ and $\left\|x_{k}-\bar{x}\right\| \leq l\left\|w_{k}-\bar{w}\right\|$ for $k>0$ sufficiently large. Hence (21) implies

$$
\begin{aligned}
\left\langle w^{*}, w_{k}-\bar{w}\right\rangle & >\mu\left(w_{k}\right)-\mu(\bar{w})+\bar{\epsilon}\left\|w_{k}-\bar{w}\right\| \\
& =f\left(x_{k}, w_{k}\right)-f(\bar{x}, \bar{w})+\bar{\epsilon}\left\|w_{k}-\bar{w}\right\| \\
& =\left\langle\nabla_{x} f(\bar{x}, \bar{w}), x_{k}-\bar{x}\right\rangle+\left\langle\nabla_{w} f(\bar{x}, \bar{w}), w_{k}-\bar{w}\right\rangle \\
& +o\left(\left\|x_{k}-\bar{x}\right\|+\left\|w_{k}-\bar{w}\right\|\right)+\bar{\epsilon}\left\|w_{k}-\bar{w}\right\| \\
& \geq\left\langle\nabla_{x} f(\bar{x}, \bar{w}), x_{k}-\bar{x}\right\rangle+\left\langle\nabla_{w} f(\bar{x}, \bar{w}), w_{k}-\bar{w}\right\rangle \\
& +o\left(\left\|x_{k}-\bar{x}\right\|+\left\|w_{k}-\bar{w}\right\|\right)+\frac{\bar{\epsilon}}{2}\left\|w_{k}-\bar{w}\right\|+\frac{l \bar{\epsilon}}{2}\left\|x_{k}-\bar{x}\right\| .
\end{aligned}
$$

Putting $\hat{\epsilon}=\min \{\bar{\epsilon} / 2, l \bar{\epsilon} / 2\}$, we get

$$
\begin{aligned}
& \left\langle\left(w^{*}-\nabla_{w} f(\bar{x}, \bar{w}),-\nabla_{x} f(\bar{x}, \bar{w})\right),\left(w_{k}-\bar{w}, x_{k}-\bar{x}\right)\right\rangle \\
& >o\left(\left\|x_{k}-\bar{x}\right\|+\left\|w_{k}-\bar{w}\right\|\right)+\hat{\epsilon}\left(\left\|w_{k}-\bar{w}\right\|+\left\|x_{k}-\bar{x}\right\|\right) .
\end{aligned}
$$

Consequently,

$$
\limsup _{(w, x) \stackrel{P \cap Q}{\longrightarrow}(\bar{w}, \bar{x})} \frac{\left\langle\left(w^{*}-\nabla_{w} f(\bar{x}, \bar{w}),-\nabla_{x} f(\bar{x}, \bar{w})\right),(w-\bar{w}, x-\bar{x})\right\rangle}{\|w-\bar{w}\|+\|x-\bar{x}\|} \geq \hat{\epsilon} .
$$

This means that

$$
\left(w^{*}-\nabla_{w} f(\bar{x}, \bar{w}),-\nabla_{x} f(\bar{x}, \bar{w})\right) \notin \widehat{N}((\bar{w}, \bar{x}) ; Q \cap P) .
$$

By Lemma 2.1 and Lemma 2.2, we have

$$
\left(w^{*}-\nabla_{w} f(\bar{x}, \bar{w}),-\nabla_{x} f(\bar{x}, \bar{w})-u^{*}\right) \notin \widehat{N}((\bar{w}, \bar{x}) ; Q)=\left\{\left(-T^{*} z^{*}, A^{*} z^{*}\right) \mid z^{*} \in Z\right\}
$$

for all $u^{*} \in \widehat{N}(\bar{x} ; K)$. From (20) we can find a vector $u^{*} \in \widehat{N}(\bar{x} ; K)$ such that $w^{*}-\nabla_{w} f(\bar{x}, \bar{w}) \in T^{*}\left[\left(A^{*}\right)^{-1}\left(\nabla_{x} f(\bar{x}, \bar{w})+u^{*}\right)\right]$. Hence there is $z^{*} \in$ $\left(A^{*}\right)^{-1}\left(\nabla_{x} f(\bar{x}, \bar{w})+w^{*}\right)$ such that $w^{*}-\nabla_{w} f(\bar{x}, \bar{w})=T^{*} z^{*}$. This implies that

$$
w^{*}-\nabla_{w} f(\bar{x}, \bar{w})=-T^{*}\left(-z^{*}\right) \text { and }-\nabla_{x} f(\bar{x}, \bar{w})-w^{*}=A^{*}\left(-z^{*}\right) .
$$


Consequently,

$\left(w^{*}-\nabla_{w} f(\bar{x}, \bar{w}),-\nabla_{x} f(\bar{x}, \bar{w})-u^{*}\right) \in \widehat{N}((\bar{w}, \bar{x}) ; Q)=\left\{\left(-T^{*} z^{*}, A^{*} z^{*}\right) \mid z^{*} \in Z\right\}$,

which contradicts to (22). Hence the second assertion is also valid and the proof of the theorem is complete.

Remark 2.1 In Theorem 2.1, if we require that $X, Y$ and $Z$ are Asplund spaces (see $[14,15]$ for the definition of Asplund spaces) and $\mathrm{imA}^{*}$ and $\mathrm{im} T^{*}$ are closed sets, then the conclusion of the theorem is still valid.

Let us give an illustrative example for Theorem 2.1.

Example 2.1 Let $X=Y=R^{2}, K=(-3,3) \times(-3,3)$,

$$
f(x, w)=\sqrt{2\left(x_{1}^{2}+x_{2}^{2}\right)}-w_{1}^{3}+w_{2}^{3}
$$

and $G(w)=\left\{\left(x_{1}, x_{2}\right) \in R^{2} \mid x_{1}+x_{2}=2 w_{1}+w_{2}\right\}$. Assume that $\bar{w}=(1,0)$ and $w^{*}=\left(w_{1}^{*}, w_{2}^{*}\right) \in \widehat{\partial} \mu(\bar{w})$. Then one has $w^{*}=(-1,1)$.

Indeed, for $\bar{w}=(1,0)$ we have

$$
\mu(\bar{w})=\inf _{\left(x_{1}, x_{2}\right) \in G(\bar{w}) \cap K}\left\{\sqrt{2\left(x_{1}^{2}+x_{2}^{2}\right)}-1\right\},
$$

where $G(\bar{w})=\left\{\left(x_{1}, x_{2}\right) \in R^{2} \mid x_{1}+x_{2}=2\right\}$. It is easy to check that $\bar{x}=(1,1)$ is the unique solution of the problem corresponding to $\bar{w}$ and $\mu(\bar{w})=1$.

Since $\bar{x} \in \operatorname{int} K, \widehat{N}(\bar{x} ; K)=\{(0,0)\}$. Hence (12) becomes

$$
\begin{aligned}
\widehat{\partial} \mu(\bar{w}) & \subset \bigcap_{\left(x^{*}, v^{*}\right) \in \hat{\partial}^{+} f(\bar{x}, \bar{w})}\left[v^{*}+T^{*}\left(\left(A^{*}\right)^{-1}\left(x^{*}\right)\right)\right] \\
& =\left\{(-3,0)+T^{*}\left(\left(A^{*}\right)^{-1}(1,1)\right)\right\}
\end{aligned}
$$

Note that

$$
A^{*}=\left[\begin{array}{l}
1 \\
1
\end{array}\right] \text { and } T^{*}=\left[\begin{array}{l}
2 \\
1
\end{array}\right] \text {. }
$$

Hence $\left.\left(A^{*}\right)^{-1}(1,1)\right)=1$ and $T^{*}\left(\left(A^{*}\right)^{-1}(1,1)\right)=(2,1)$. Combining this with $(23)$, we get $\widehat{\partial} \mu(\bar{w}) \subset\{(-1,1)\}$. By computing directly, we see that 
$x=\left(\frac{2 w_{1}+w_{2}}{2}, \frac{2 w_{1}+w_{2}}{2}\right)$ is a unique solution of the problem corresponding to $w=\left(w_{1}, w_{2}\right)$ in a neighborhood of $\bar{w}$. Thus the solution map is Lipschitz continuous on a neighborhood of $\bar{w}$. Hence we obtain $\hat{\partial} \mu(\bar{w})=\{(-1,1)\}$.

\section{$3 \quad$ Proof of Theorem 1.1}

In what follows, we will formulate the problem (1)-(4) in the form of the problem (10)-(11) and use the abstract result in section 2 to finish the proof.

Put $Z=X \times U, K=X \times \Omega, Y=X_{1} \times X_{2} \times \cdots \times X_{N}$. Then the problem (1)-(4) can be written as the following form:

$$
V(w)=\inf _{z \in G(w) \cap K} f(z, w),
$$

where

$$
G(w)=\{z=(x, u) \in Z \mid A z=T w\},
$$

$A: Z \rightarrow Y$ and $T: W \rightarrow Y$ are defined, respectively, by

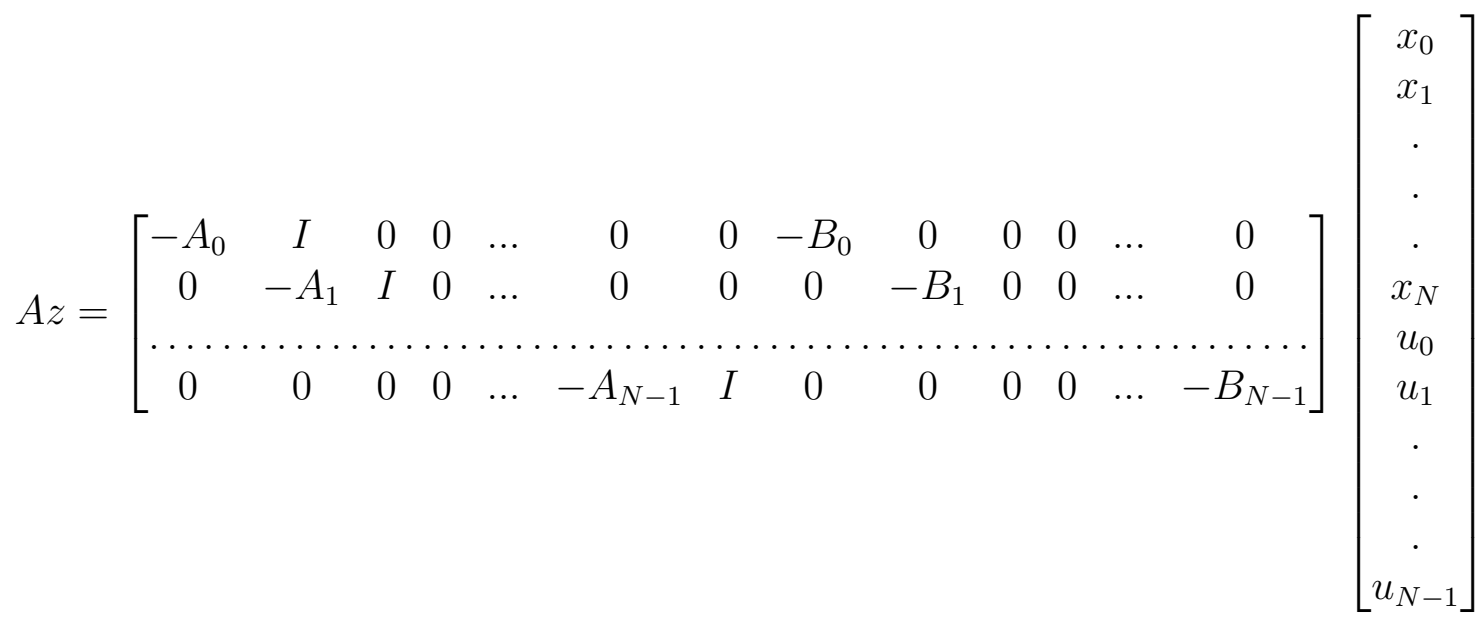

$$
\begin{aligned}
& T w=\left[\begin{array}{c}
T_{0} w_{0} \\
T_{1} w_{1} \\
\cdot \\
\cdot \\
\cdot \\
T_{N-1} w_{N-1}
\end{array}\right]
\end{aligned}
$$


According to Theorem 2.1, it follows that if $w^{*} \in \widehat{\partial} V(\bar{w})$ then there exist a vector $u^{*} \in \widehat{N}(\bar{z} ; K)$ and $z^{*}=\left(z_{1}^{*}, z_{2}^{*}, \ldots, z_{N}^{*}\right) \in Y$ such that

$$
w^{*}=\nabla_{w} f(\bar{z}, \bar{w})+T^{*} z^{*} \text { and } \nabla_{z} f(\bar{z}, \bar{w})+u^{*}=A^{*} z^{*} .
$$

It remains to compute $\nabla_{w} f(\bar{z}, \bar{w}), \nabla_{z} f(\bar{z}, \bar{w}), T^{*} z *$ and $A^{*} z^{*}$.

Step 1 (Computation of $\nabla_{w} f(\bar{z}, \bar{w})$ and $\nabla_{z} f(\bar{z}, \bar{w})$ ). Since

$$
f(z, w)=f(x, u, w)=\sum_{k=0}^{N-1} h_{k}\left(x_{k}, u_{k}, w_{k}\right)+h_{N}\left(x_{N}\right),
$$

we have

$$
\begin{aligned}
& \nabla_{w} f(\bar{x}, \bar{u}, \bar{w})=\sum_{k=0}^{N-1} \nabla_{w} h_{k}\left(x_{k}, u_{k}, w_{k}\right) \\
& =\left(\frac{\partial h_{0}}{\partial w_{0}}\left(\bar{x}_{0}, \bar{u}_{0}, \bar{w}_{0}\right), \frac{\partial h_{0}}{\partial w_{1}}\left(\bar{x}_{1}, \bar{u}_{1}, \bar{w}_{1}\right), \ldots, \frac{\partial h_{N-1}}{\partial w_{N-1}}\left(\bar{x}_{N-1}, \bar{u}_{N-1}, \bar{w}_{N-1}\right)\right) .
\end{aligned}
$$

Also, we get

$$
\begin{aligned}
& \nabla_{z} f(\bar{x}, \bar{u}, \bar{w})=\sum_{k=0}^{N-1}\left(\nabla_{x} h_{k}(\bar{x}, \bar{u}, \bar{w}), \nabla_{u} h_{k}(\bar{x}, \bar{u}, \bar{w})\right) \\
= & \left(\frac{\partial h_{0}}{\partial x_{0}}\left(\bar{x}_{0}, \bar{u}_{0}, \bar{w}_{0}\right), \ldots, \frac{\partial h_{N-1}}{\partial x_{N-1}}\left(\bar{x}_{N-1}, \bar{u}_{N-1}, \bar{w}_{N-1}\right), \frac{\partial h_{N}}{\partial x_{N}}\left(\bar{x}_{N}\right) ;\right. \\
& \left.\frac{\partial h_{0}}{\partial u_{0}}\left(\bar{x}_{0}, \bar{u}_{0}, \bar{w}_{0}\right), \ldots, \frac{\partial h_{N-1}}{\partial u_{N-1}}\left(\bar{x}_{N-1}, \bar{u}_{N-1}, \bar{w}_{N-1}\right)\right) .
\end{aligned}
$$

Step 2 (Computation of $T^{*} z^{*}$ and $A^{*} z^{*}$ ). It is easy to verify that $T^{*} z^{*}=$ $\left[T_{0}^{*} z_{1}^{*}, T_{1}^{*} z_{2}^{*}, \ldots, T_{N-1}^{*} z_{N}^{*}\right]$. From the formula of $A$, we get

$$
A^{*} z^{*}=\left[\begin{array}{ccccc}
-A_{0}^{*} & 0 & 0 & \ldots & 0 \\
I & -A_{1}^{*} & 0 & \ldots & 0 \\
0 & I & 0 & \ldots & 0 \\
\ldots \ldots & \ldots & \ldots & \ldots \ldots & \ldots \\
0 & 0 & 0 & \ldots & -A_{N-1}^{*} \\
0 & 0 & 0 & \ldots & I \\
-B_{0}^{*} & 0 & 0 & \ldots & 0 \\
0 & -B_{1}^{*} & 0 & \ldots & 0 \\
\ldots \ldots & \ldots & \ldots \\
0 & 0 & 0 & \ldots & -B_{N-1}^{*}
\end{array}\right]\left[\begin{array}{c}
z_{1}^{*} \\
z_{2}^{*} \\
\cdot \\
\cdot \\
\cdot \\
z_{N}^{*}
\end{array}\right]
$$


Note that $\widehat{N}(\bar{z} ; K)=\{0\} \times \widehat{N}(\bar{u} ; \Omega)$. Hence $u^{*}=\left(0, \bar{u}^{*}\right)$ for some $\bar{u}^{*}=$ $\left(u_{0}^{*}, u_{1}^{*}, \ldots, u_{N-1}^{*}\right)$, where $u_{k}^{*} \in \widehat{N}\left(\bar{u}_{k} ; \Omega_{k}\right)$ for $k=0,1, \ldots, N-1$.

We now substitute $\left.\nabla_{w} f(\bar{z}, \bar{w}), \nabla_{z} f(\bar{z}, \bar{w})\right), T^{*} z^{*}, A^{*} z^{*}$ and $u^{*}=\left(0, \bar{u}^{*}\right)$ into (24). From the first equation of (24), we obtain

$$
w_{k}^{*}=\frac{\partial h_{k}}{\partial w_{k}}\left(\bar{x}_{k}, \bar{u}_{k}, \bar{w}_{k}\right)+T_{k}^{*} z_{k}^{*} \text { for } k=0,1, \ldots, N-1 .
$$

The second equation of (24) gives

$$
\begin{gathered}
\left(\frac{\partial h_{0}}{\partial x_{0}}\left(\bar{x}_{0}, \bar{u}_{0}, \bar{w}_{0}\right), \ldots, \frac{\partial h_{N-1}}{\partial x_{N-1}}\left(\bar{x}_{N-1}, \bar{u}_{N-1}, \bar{w}_{N-1}\right), \frac{\partial h_{N}}{\partial x_{N}}\left(\bar{x}_{N}\right)\right)= \\
=\left[\begin{array}{ccccc}
-A_{0}^{*} & 0 & 0 & \ldots & 0 \\
I & -A_{1}^{*} & 0 & \ldots & 0 \\
0 & I & -A_{2}^{*} & \ldots & 0 \\
\ldots \ldots & \ldots & \ldots & \ldots \\
0 & 0 & 0 & \ldots & -A_{N-1}^{*} \\
0 & 0 & 0 & \ldots & I
\end{array}\right]\left[\begin{array}{c}
z_{1}^{*} \\
z_{2}^{*} \\
\cdot \\
\cdot \\
. \\
z_{N}^{*}
\end{array}\right]
\end{gathered}
$$

and

$$
\begin{gathered}
\left(\frac{\partial h_{0}}{\partial u_{0}}\left(\bar{x}_{0}, \bar{u}_{0}, \bar{w}_{0}\right), \ldots, \frac{\partial h_{N-1}}{\partial u_{N-1}}\left(\bar{x}_{N-1}, \bar{u}_{N-1}, \bar{w}_{N-1}\right)\right)+u^{*}= \\
=\left[\begin{array}{ccccc}
-B_{0}^{*} & 0 & 0 & \ldots & 0 \\
0 & -B_{1}^{*} & 0 & \ldots & 0 \\
\ldots \ldots & \ldots \ldots \ldots \ldots \ldots \ldots \ldots \\
0 & 0 & 0 & \ldots & -B_{N-1}^{*}
\end{array}\right]\left[\begin{array}{c}
z_{1}^{*} \\
z_{2}^{*} \\
\cdot \\
\cdot \\
\cdot \\
z_{N}^{*}
\end{array}\right]
\end{gathered}
$$

Hence from equation (25), we have

$$
\left\{\begin{array}{l}
\frac{\partial h_{0}}{\partial x_{0}}\left(\bar{x}_{0}, \bar{u}_{0}, \bar{w}_{0}\right)=-A_{0}^{*} z_{1}^{*} \\
\frac{\partial h_{1}}{\partial x_{1}}\left(\bar{x}_{1}, \bar{u}_{1}, \bar{w}_{1}\right)=z_{1}^{*}-A_{1}^{*} z_{2}^{*} \\
\cdots \cdots \ldots . \cdots \\
\frac{\partial h_{N-1}}{\partial x_{N-1}}\left(\bar{x}_{N-1}, \bar{u}_{N-1}, \bar{w}_{N-1}\right)=z_{N-1}^{*}-A_{N-1}^{*} z_{N}^{*} \\
\left.\frac{\partial h_{N}}{\partial x_{N}}\left(\bar{x}_{N}\right)\right)=z_{N}^{*} .
\end{array}\right.
$$


Also (26) implies $\frac{\partial h_{k}}{\partial u_{k}}\left(\bar{x}_{k}, \bar{u}_{k}, \bar{w}_{k}\right)=-u_{k}^{*}-B_{k}^{*} z_{k+1}^{*}$ for $k=0,1, \ldots, N-1$. Thus the first assertion of Theorem 1.1 is valid.

If the solution map $S$ has an upper Lipschitzian selection at $(\bar{w}, \bar{x}, \bar{u})$, then it is also a sufficient condition for $w^{*} \in \widehat{\partial} V(\bar{w})$ because of the second assertion of Theorem 2.1. The proof of the theorem is complete.

Let us give an illustrative example for Theorem 1.1.

Example 3.1. Consider the problem

$$
\left\{\begin{aligned}
f(x, u, w) & =\Sigma_{k=0}^{2}\left(\left(x_{k}+u_{k}-w_{k}\right)^{2}-w_{k}\right)+\frac{1}{1+x_{3}^{2}} \rightarrow \inf \\
x_{k+1} & =x_{k}+u_{k}-w_{k}, k=0,1,2 . \\
x(0) & =1 .
\end{aligned}\right.
$$

Let $\bar{w}=\left(\bar{w}_{0}, \bar{w}_{1}, \bar{w}_{2}\right)=(0,0,0)$ and $w^{*} \in \widehat{\partial} V(\bar{w})$. Then one has $w^{*}=$ $(-1,-1,-1)$.

In fact, when $\bar{w}=(0,0,0)$ the problem becomes

$$
\left\{\begin{aligned}
f(x, u, \bar{w}) & =\Sigma_{k=0}^{2}\left(x_{k}+u_{k}\right)^{2}+\frac{1}{1+x_{3}^{2}} \rightarrow \min \\
x_{k+1} & =g\left(x_{k}, u_{k}, k\right):=x_{k}+u_{k}, k=0,1,2 . \\
x_{0} & =1 .
\end{aligned}\right.
$$

Put $\left.I(x, i)=\min \Sigma_{k=i}^{2}\left(x_{k}+u_{k}\right)\right)^{2}+\frac{1}{1+x_{3}^{2}}$, where

$$
\left\{\begin{array}{l}
x_{k+1}=g\left(x_{k}, u_{k}, k\right)=x_{k}+u_{k}, k=i, \ldots, 2 . \\
x_{i}=x
\end{array}\right.
$$

According to Bellman equation, we have $I(x, 3)=\frac{1}{1+x^{2}}$,

$$
\begin{aligned}
I(x, 2) & =\min _{u}\left[(x+u)^{2}+I(g(x, u, 2), 3)\right] \\
& =\min _{u}\left[(x+u)^{2}+\frac{1}{1+(x+u)^{2}}\right] \\
& =1 .
\end{aligned}
$$

The optimal value $I(x, 2)=1$ is obtained at $u=-x$. Hence

$$
\begin{aligned}
I(x, 1) & =\min _{u}\left[(x+u)^{2}+I(g(x, u, 1), 2)\right] \\
& =\min _{u}\left[(x+u)^{2}+1\right]=1 \text { at } u=-x .
\end{aligned}
$$


Finally, we get

$$
\begin{aligned}
I(x, 0) & =\min _{u}\left[(x+u)^{2}+I(g(x, u, 0), 1)\right] \\
& =\min _{u}\left[(x+u)^{2}+1\right]=1 \text { at } u=-x .
\end{aligned}
$$

Since $x_{0}=1$, we have $u_{0}=-x_{0}=-1$. Hence $x_{1}=x_{0}+u_{0}=0$ and $u_{1}=-x_{1}=0$. Similarly, we have $x_{2}=x_{1}+u_{1}=0, u_{2}=-x_{2}=0$ and $x_{3}=x_{2}+u_{2}=0$. Thus the couple of $\bar{x}=(1,0,0,0)$ and $\bar{w}=(-1,0,0)$ is the unique solution of the problem corresponding to $\bar{w}=(0,0,0)$. We now have, $h_{k}\left(x_{k}, u_{k}, w_{k}\right)=\left(x_{k}+u_{k}-w_{k}\right)^{2}-w_{k}$ with $k=0,1,2$, and $h_{3}\left(x_{3}\right)=\frac{1}{1+x_{3}^{2}}$. By computing, we get $\left(\frac{\partial h_{3}}{\partial x_{3}}\right)\left(\bar{x}_{3}\right)=0,\left(\frac{\partial h_{k}}{\partial x_{k}}\right)\left(\bar{x}_{k}, \bar{u}_{k}, \bar{w}_{k}\right)=0,\left(\frac{\partial h_{k}}{\partial u_{k}}\right)\left(\bar{x}_{k}, \bar{u}_{k}, \bar{w}_{k}\right)=0$ and $\left(\frac{\partial h_{k}}{\partial w_{k}}\right)\left(\bar{x}_{k}, \bar{u}_{k}, \bar{w}_{k}\right)=-1$ for all $k=0,1,2$. For this problem, one has $A_{0}=A_{1}=A_{2}=1, B_{0}=B_{1}=B_{2}=1$ and $T_{0}=T_{1}=T_{2}=-1$. By Corollary 1.1, there exist $z_{1}^{*}, z_{2}^{*}$ and $z_{3}^{*}$ such that

$$
\left\{\begin{array}{l}
w_{k}^{*}=\left(\frac{\partial h_{k}}{\partial w_{k}}\right)\left(\bar{x}_{k}, \bar{u}_{k}, \bar{w}_{k}\right)+T_{k}^{*} z_{k+1}^{*} \text { for } k=0,1,2 . \\
\left(\frac{\partial h_{0}}{\partial x_{0}}\right)\left(\bar{x}_{0}, \bar{u}_{0}, \bar{w}_{0}\right)=-A_{0}^{*} z_{1}^{*},\left(\frac{\partial h_{3}}{\partial x_{3}}\right)\left(\bar{x}_{3}\right)=z_{3}^{*}, \\
\left(\frac{\partial h_{1}}{\partial x_{1}}\right)\left(\bar{x}_{1}, \bar{u}_{1}, \bar{w}_{1}\right)=z_{1}^{*}-A_{1}^{*} z_{2}^{*} \\
\left(\frac{\partial h_{2}}{\partial x_{2}}\right)\left(\bar{x}_{2}, \bar{u}_{2}, \bar{w}_{2}\right)=z_{2}^{*}-A_{2}^{*} z_{3}^{*} \\
\left(\frac{\partial h_{0}}{\partial u_{0}}\right)\left(\bar{x}_{0}, \bar{u}_{0}, \bar{w}_{0}\right)=-B_{0}^{*} z_{1}^{*} \\
\left(\frac{\partial h_{1}}{\partial u_{1}}\right)\left(\bar{x}_{1}, \bar{u}_{1}, \bar{w}_{1}\right)=-B_{1}^{*} z_{2}^{*} \\
\left(\frac{\partial h_{2}}{\partial u_{2}}\right)\left(\bar{x}_{2}, \bar{u}_{2}, \bar{w}_{2}\right)=-B_{2}^{*} z_{3}^{*} .
\end{array}\right.
$$

Hence $z_{1}^{*}=z_{1}^{*}=z_{3}^{*}=0$ and $w_{0}^{*}=w_{1}^{*}=w_{2}^{*}=-1$. Thus $\widehat{\partial} V(\bar{w}) \subseteq$ $\{(-1,-1,-1)\}$. By computing we see that the couple of $x=\left(1, w_{0}, w_{1}, w_{2}\right)$ and $u=\left(w_{0}-1, w_{1}-w_{0}, w_{2}-w_{1}\right)$ is the unique solution of the problem corresponding to the parameter $w=\left(w_{0}, w_{1}, w_{2}\right)$. Thus the solution map is Lipschitz continuous. By the second assertion of Theorem 1.1, we have $\widehat{\partial} V(\bar{w})=\{(-1,-1,-1)\}$.

Acknowledgements The authors wish to to express their sincere thanks to the referees for many helpful suggestions and comments which improve the original manuscript greatly.

\section{References}

[1] J.-P.Aubin and I. Ekeland, Applied Nonlinear Analysis, Wiley, New York, 1984. 
[2] A. V. Arutyunov and B. Marinkovich, Necessary optimality conditions for discrete optimal control problems, Mosc. Univ. Comput. Math. Cybern., 1, 38-44, 2005.

[3] D. P. Bertsekas, Dynamic Programming and Optimal Control, Vol. I, Springer, 2005.

[4] J.M. Borwein and Qiji J. Zhu, Techniques of Variational Analysis, Springer, New York, 2005.

[5] F. H. Clarke, Method of Dynamic and Nonsmooth Optimization, Philadelphia, 1989.

[6] F. H. Clarke Optimization and Nonsmooth Analysis, SIAM, Philadelphia, 1990.

[7] R. Gabasov, B. Mordukhovich and F. M. Kirillova, The discrete maximum principle, Dokl. Akad. Nauk SSSR, 213, 19-22, 1973. (Russian; English transl. in Soviet Math. Dokl. 14, 1624-1627, 1973).

[8] A. Ioffe, Euler-Lagrange and Hamiltonian formalisms in dynamic optimization, Tran. AMS., 349(1997), pp. 2871-2900.

[9] R. E. Larson and J. Casti, Principles of Dynamic Programming, Vol. I, Marcel Dekker, 1982.

[10] R. E. Larson and J. Casti, Principles of Dynamic Programming, Vol. II, Marcel Dekker, 1982.

[11] Z. Lian, L. Liu, and M.F. Neuts, A discrete-time model for common lifetime inventory systems, Math. Oper. Res., 30, 718-732, 2005.

[12] S. E. Lyshevski, Control system theory with engineering applications, Control Engineering, Birkhäuser, Boston, MA, 2001.

[13] V. N. Malozemov and A. V. Omelchenko, On a discrete optimal control problem with an explicit solution, J. Ind. Manag. Optim. 2, 55-62, 2006.

[14] B. S. Mordukhovich, Variational Analysis and Generalized Differentiation I, Basis Theory, Springer, 2006.

[15] B. S. Mordukhovich, Variational Analysis and Generalized Differentiation II, Applications, Springer, 2006.

[16] B. S. Mordukhovich, N. M. Nam and N. D. Yen, Subgradients of marginal 
functions in parametric mathematical programming, Math. Program. (to appear) 2006.

[17] B. S. Mordukhovich and N. M. Nam and N. D. Yen, Fréchet subdifferential calculus and optimality conditions in nondifferetiable programming, Optimization 55, 685-708, 2006.

[18] B. S. Mordukhovich and N. M. Nam, Variational stability and marginal functions via generalized differentiation, Math. Oper. Res., 30, 800-816, 2005.

[19] B.S. Mordukhovich, Difference approximations of optimal control system, Prikl. Mat. Mekh., 42, 431-440, 1978. ( Russian; English transl. in J. Appl. Math. Mech., 42, 452-461, 1978).

[20] M. Moussaoui and A. Seeger, Sensitivity analysis of optimal value functions of convex parametric programs with possibly empty solution sets, SIAM J. Optim, 4, 659-675, 1994.

[21] J.-P. Penot, Differetiability properties of optimal value functions, Canadian J. Math., 56(4), 825-842, 2004.

[22] R. T. Rockafellar, Hamilton-Jacobi theory and parametric analysis in fully convex problems of optimal control, J. Global Optim. 248, 419-431, 2004.

[23] R. T. Rockafellar and P. R. Wolenski, Convexity in Hamilton-Jacobi theory I: dynamics and duality, SIAM J. Control Optim. 39, 1323-1350, 2000 .

[24] A. Seeger, Subgradient of optimal-value function in dynamic programming: The case of convex system without optimal paths, Math. Oper. Research, 21, 555-575, 1996.

[25] R. B. Vinter, Optimal Control, Birkhäuser, Bostn 2000.

[26] R. B. Vinter and H. Zheng, Necessary conditions for optimal control problems with state constraints, Tran. AMS, 350, 1181-1204, 1998. 